\title{
A Review on Intelligent Traffic with Emergency Control and Stolen Vehicle Tracking System
}

\author{
Arun Pratap Singh \\ M.Tech. CSE, IV Semester \\ LIG-46, Harshwardhan Nagar, Bhopal, \\ M.P. 462003
}

\author{
Sanjay Kumar Sharma \\ Associate Professor \\ Oriental Institute of Science \& Technology, \\ Bhopal
}

\begin{abstract}
The motive of this paper is to design and implement "Intelligent Traffic with Emergency Control and Stolen Vehicle Tracking System" using Artificial Intelligence. Thus this system is capable to sense the density of vehicles for intelligent traffic management and also able to recognize emergency vehicles as ambulance to allow that signal for emergency control, as well as this system also recognizes the vehicles number for tracking stolen vehicles. It is significant to identify the density of traffic on real time mainly in metropolitan cities for signals control and active traffic management using traffic mass estimation using neural networks. In Density Control System we allow that signal first which has the highest density of vehicles, through which the congestion of traffics can be controlled then signals will get back to its original position or flow. In Emergency Control System we recognize the ambulance using pattern recognition among other vehicles from traffic and if system recognized emergency vehicle then that particular signal will be allowed and other signals will become red and an emergency alarm will be generated to indicate the activation of emergency signal. And in Stolen Vehicle Tracking System, our system will recognize vehicles numbers from those vehicles which are waiting for green signal then it will verify the validation and authentication of that vehicles numbers and report the location of signal to the control room if any fraudulent information is found. This entire system is based on artificial intelligence that can make better control system.
\end{abstract}

\section{Keywords}

Pattern Recognition, Neural Network, Density Control System, Stolen Vehicle Tracking System, Emergency Control System, MATLAB etc.

\section{INTRODUCTION}

Today's Traffic Controllers are based on microcontrollers and microprocessors. These Traffic Controllers have some restrictions because it uses the predefined hardware and software, which is working according to the program that doesn't have flexibility of decision making, where there is no emergency control as well as tracking system.

Several other sensors such as radar, infrared and microwave etc. detectors exist. These sensors are costly with limited capability and comprise installation, maintenance, and implementation problems. In modern era, video processing techniques have involved for vehicle detection.
But our system recognizes ambulance for emergency control which turns all signals red except that signal where that ambulance being stuck.

Our system also recognizes the density of vehicles for effective traffic management, in which highest vehicles density lane will get the priority and then the system will work in traditional way, after each interval of time we check the density of vehicles to minimize the congestion of traffics during rush hours.

It also recognizes the vehicles number for tracking stolen vehicles by finding and verifying that vehicles number from its data base which contains a list of complaints of stolen vehicles and inform the nearest control room if match found, and also if vehicle number does not exist in data base of RTO in case of replaced number plates then it also inform about the fraudulent to the nearest control room with their signals location of that particular area.

\section{BASE PAPERS}

The base paper uses the RF transmitters and receivers to track the ambulance for emergency control and any vehicle if stolen. But the drawback of this system is that it doesn't have the wide range of recognition or we can say that it has limited range of recognition to track any vehicle and we also need to install this system in each vehicle as well as in each traffic signal. So, it is not possible to install the system in each and every vehicle including emergency vehicles. It is also not cost effective.

To replace this system another concept has been introduced that is Global Positioning System(GPS) to track the ambulances for emergency control and any vehicle if stolen which has wide range of recognition. But it has also some drawbacks that is we also need to install this Global Positioning System or GPS in each vehicle as well as in each traffic signal and this system module requires energy source/battery power. So, that's why it is also not cost effective.

To overcome this problem another paper has been proposed which does not require battery, it uses green wave receiver but all these system stuck at the same point that is it also have to install in each and every vehicle as well as in each traffic signal which is also not cost effective. 


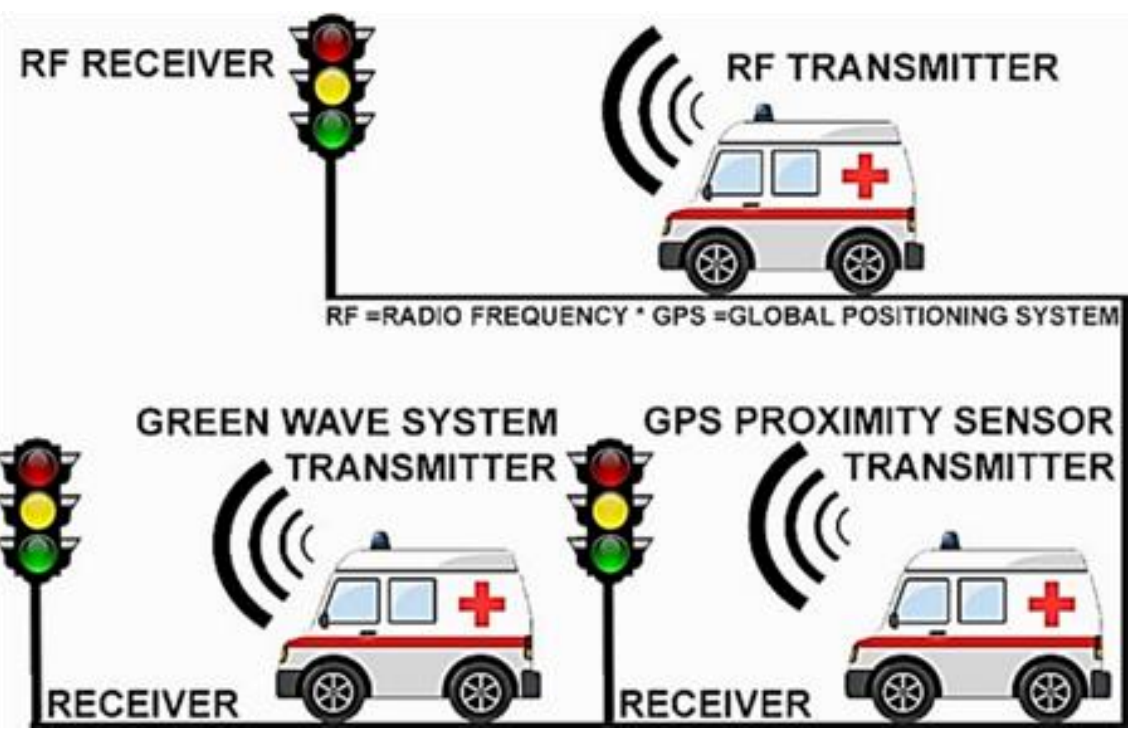

Fig. 2.1 Base Systems (RF, GPS, GWR)

\section{PROPOSED PAPER}

To overcome the above problems we developed a new promising concept which follows the pattern recognition techniques. This proposed method is cost effective because we do not need to install any kind of receiver in the vehicles. Here the problem is divided into three sub-problems.

\subsection{Emergency Control System}

In this system, a high resolution CCTV cameras will recognize the ambulances as well as vehicle numbers by using pattern recognition.

By recognizing an ambulance, the system will make some changes in traffic signal which turns signal red at all sides except that side where the ambulance was being stuck, it means, that side will get the green signal which helps to save human lives which may be in critical condition.

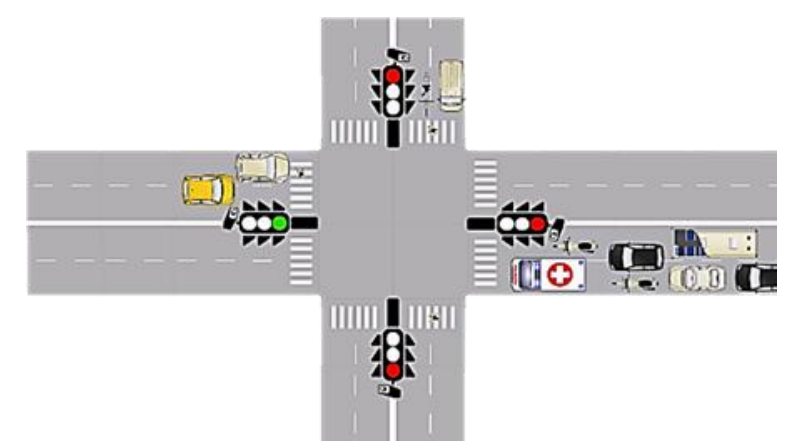

Fig. 3.1 Emergency Control System

\subsection{Stolen Vehicle Tracking System}

It also recognizes the vehicle numbers from those vehicles which are waiting for green signal and after recognition it helps to catch stolen vehicles.

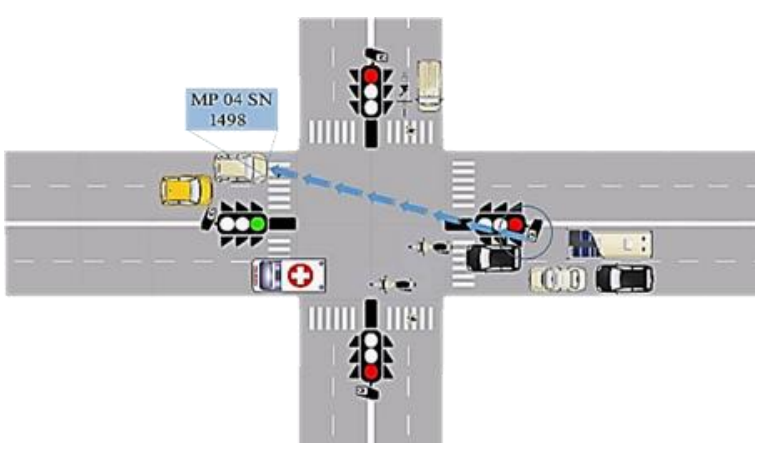

Fig.3.2 Stolen Vehicle Tracking System

\subsection{Density Control System}

This system also provides density control features by this we can manage congestions during rush hours.

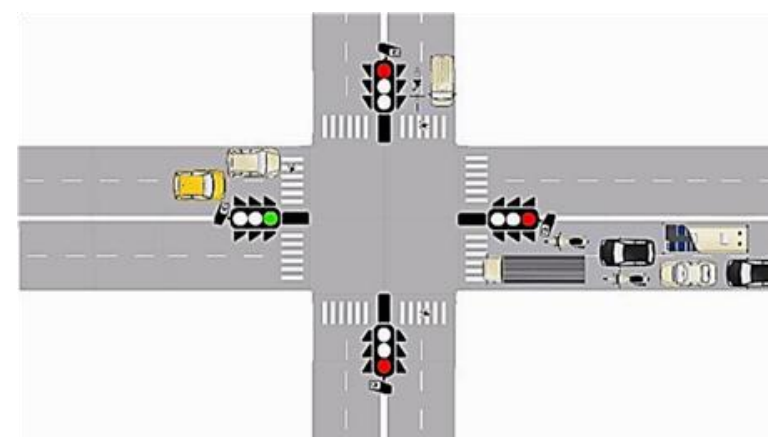

Fig. 3.3 Density Control System

\section{CONTROL STRATEGY}

Here the problem is divided into three sub problems, first one is Density Control, second one is Emergency Control and third one is Stolen Vehicle Tracking System. This system can be achieved by pattern recognition using supervised learning in artificial intelligence.

Pattern recognition technology makes it possible to identify objects, signals, persons etc. with a high level of accuracy.

It is significant to identify the traffic density for intelligent traffic management. Now a day's video monitoring systems have been largely used in traffic management. In this system camera is mounted on the top of the signal to get the wide 
angled view of traffic on the each side of the signal so that it will analyze the traffic in that particular side and count of the numbers of vehicles. With the count of vehicles the density of that particular side will be determined and corresponding signal will be provided.

\section{VEHICLES FOUND}

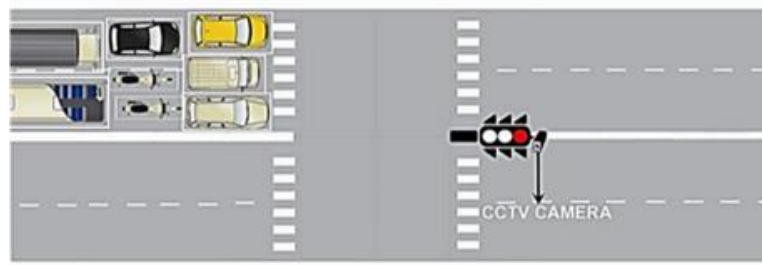

DENSITY CONTROL SYSTEM

Fig. 4.1 Density Control System

In today's world, traffic jams during rush hours is one of the major problems. During rush hours, emergency vehicles i.e. Ambulances get stuck in jams. Due to that, emergency vehicles i.e. Ambulances are not able to reach hospital on time, resulting loss of human lives.

To tackle this situation we developed an Emergency Control System. In an emergency control system we use pattern recognition technology by which we will be able to identify the ambulance among the vehicles in the traffic. Some standard signs and combination of letters are unique in ambulances like

\section{†,ヨИА」UЯMA,AMBULANCE}

Our system recognizes these standards by using high definition CCTV camera to identify the ambulances in traffic. By recognizing an ambulance, the system will make some changes in traffic signal which turns signal red at all sides except that side where the ambulance was being stuck, it means, that side will get the green signal which helps to save human lives which may be in critical condition.

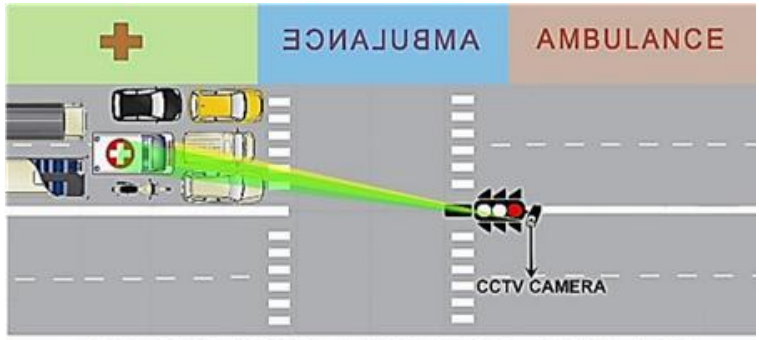

EMERGENCY CONTROL SYSTEM

Fig. 4.2 Emergency Control System

In Stolen Vehicle Tracking System, there will be a high resolution CCTV camera mounted around each pole of traffic signals. Those CCTV cameras will be capable to recognize vehicle numbers automatically.

Each CCTV camera recognizes the vehicles number from those vehicles which are waiting for green signal and after recognition the recognized vehicles number will be verified with those stolen vehicle's number which were registered at police station by their owners. If match found then it will be informed to the nearest control room about the signal's location of that particular area with their vehicle number, which helps to catch the stolen vehicles.

This system follows the different conditions to be satisfied if any one of the given condition will be true -

- If recognized vehicle number does not exist.

- If recognized vehicle number is found in complaint list.

In all above conditions the same action will be conducted as specified earlier.

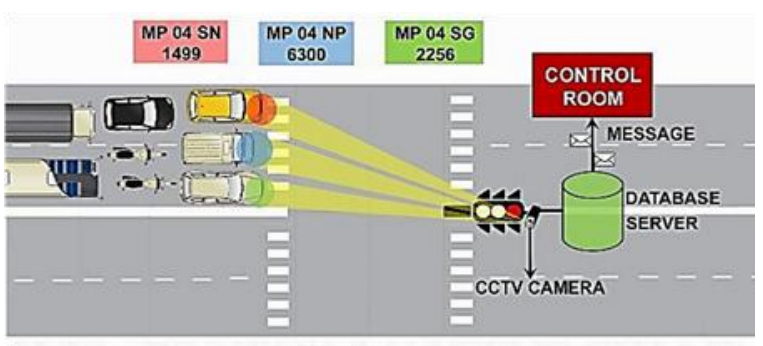

STOLEN VEHICLE TRACKING SYSTEM

Fig. 4.3 Stolen Vehicle Tracking System

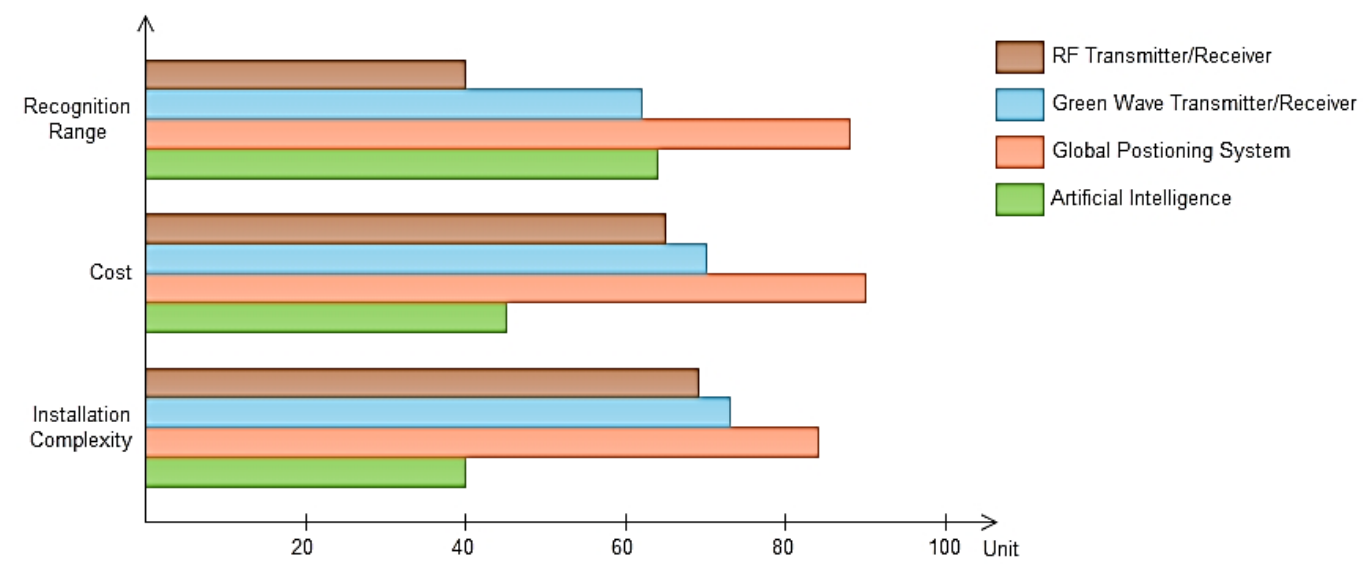

Fig. 4.4 Compared Result Analysis 


\section{FUTURE SCOPE}

The current proposed concept of artificial intelligence to replace the GPS system as well as RF transmitter in order to overcome the complexity of placing transmitter in each vehicle as well as in signal and to make the system cost effective. This system can save human lives as well as can track stolen vehicles.

\section{CONCLUSION}

Thus the real time implementation of proposed concept of implementing recognizing system for ambulances could ease the difficulties faced during jams by ambulances when reaching to the hospital or to the injured or accidental spot. It also minimize the congestion of traffic during rush hour for effective traffic management and control. It may also track stolen vehicle which reduces the human effort.

\section{REFERENCES}

[1] Shilpa S. Chavan (Walke) 1, Dr. R. S. Deshpande3, J. G. Rana2 proposed Design Of Intelligent Traffic Light Controller Using Embedded System at Second International Conference on Emerging Trends in Engineering and Technology, ICETET-09 which is based on GSM technology.

[2] Sarika B.Kale proposed Embedded system for intelligent Ambulance and traffic control Management at International Journal of Computer and Electronics Research [Volume 2, Issue 2, April 2013] which is based on RFID.

[3] Sachin Jaiswal, Tushar Agarwal, Akanksha Singh and Lakshita proposed Intelligent Traffic Control Unit at International Journal of Electrical, Electronics and Computer Engineering 2(2): 66-72(2013) which is also based on RF transmitters and Receivers.
[4] Manoj Prabhakar K and Manoj Kumar S proposed GPS Tracking System Coupled With Image Processing In Traffic Signals to Enhance Life Security at International Journal of Computer Science \& Information Technology Vol 5, No 4, August 2013 which is based on image processing and GPS system.

[5] K.Sangeetha1, P.Archana2 , M.Ramya3 , P.Ramya4 proposed an Automatic Ambulance Rescue With Intelligent Traffic Light System at IOSR Journal of Engineering (IOSRJEN) Vol. 04, Issue 02 (February. 2014) which is also based on GPS system.

[6] 1, Jyoti Sharma, 2, Savita Sivani proposed LAN Based Traffic light control System with Emergency service Identification \& Density Based Control at International Journal of Engineering Science Invention || Volume 3 || Issue 6 || June 2014 which is based on LAN.

[7] Farheena Shaikh1, Dr. Prof. M. B. Chandak2 proposed An Approach towards Traffic Management System using Density Calculation and Emergency Vehicle Alert at International Conference on Advances in Engineering \& Technology -2014 which is based GPS system.

[8] M. Alyürük, Information Technologies Chief at Istanbul Traffic Management Company (ISBAK), Inteview on June 2008.

[9] X. Ji, Z. Wei, Y. Feng, Effective vehicle detection techniques for traffic surveillence systems, J. Vis. Commun. Image R. 17, 647-658, 2006

[10] J. Zhou, D. Gao, D. Zhang, Moving vehicle detection for automatic traffic monitoring, IEEE Trans. Vehic. Tech., 56(1), 5159, 2007. 